\title{
EFECTO DE LA FERTILIZACIÓN SOBRE ALGUNOS PARÁMETROS FÍSICOS Y QUÍMICOS DE SUELOS ESTABLECIDO CON PASTURA ANGLETÓN (Dichantium aristatum) EN EL MUNICIPIO DE TOLÚ - SUCRE-COLOMBIA
}

\section{EFFECT OF FERTILIZATION ON SOME PHYSICAL AND CHEMICAL PARAMETERS WITH ESTABLISHED PASTURE SOIL ANGLETON (Dichanthium aristatum) IN THE MUNICIPALITY OF TOLÚ - SUCRE-COLOMBIA}

\author{
NAVARRO, M. ORLANDO ${ }^{1}$ MSc., PÉREZ, C. RICARDO ${ }^{2}$ MSc., CONSUEGRA, \\ D. CARLOS ${ }^{3}$ I. Agrícola., SEVERICHE, P. MARÍA ESTELLA ${ }^{3}$ I. Agrícola.
}

${ }^{1}$ Docente, Facultad de Ciencias Agropecuarias, ${ }^{2}$ Docente, Facultad de Ciencias Agropecuarias, ${ }^{3}$ Ingenieros Agrícolas, Universidad de Sucre, Colombia.

Correspondencia: ornameunisucre@yahoo.com

Recibido: 29-11-2013; Aceptado: 19-05-2014.

\section{Resumen}

El estudio se llevó a cabo en suelos de fincas ganaderas ubicadas en el corregimiento Pita Abajo, municipio de Tolú, departamento de Sucre, establecidas con pasto angletón; el objetivo de este trabajo fue el de evaluar los efectos de la fertilización química y orgánica sobre algunas propiedades físicas y químicas de un suelo con características vérticas. Se utilizó un diseño de bloques completamente al azar con cinco tratamientos y tres repeticiones, que consistieron en: Testigo absoluto $\left(T_{0}\right)$, Urea $\left(T_{1}\right)$, Urea + Lombricompost $\left(T_{2}\right)$, Lombricompost $\left(T_{3}\right)$, y Bovinaza fresca en estado liquido $\left(T_{4}\right)$. humedad, densidad aparente, porosidad total, infiltración, materia orgánica, $\mathrm{pH}$ y capacidad de intercambio catiónico. El tratamiento Lombricompost, presentó las mejores condiciones físicas y químicas edáficas, mientras que en los tratamientos químico y testigo se dieron las condiciones físicas menos favorables, presentando también el químico la mayor acidez, y el tratamiento bovinaza líquida el menor contenido de materia orgánica y la más baja capacidad de intercambio catiónico.

Palabras clave: fertilización, físico-químicas, abonos orgánicos, Dichanthium aristatum.

\section{Abstract}

In a soil with vertics characteristics in the Casanare property located in the corregimient Down Pita municipality of Tolú Department of Sucre where is established a crop of Angleton (Dichantium aristatum) grass, realized this test with in order to evaluate the effects of the chemical and organic fertilization and the 
incidence of the grass on some physical and chemical properties of the soil, using an experimental design completely at random with five treatments and three repetitions, that they consisted of a witness $\left(T_{0}\right)$ to whom was not done no application, application of Urea $\left(T_{1}\right)$, application of Urea + Lombricompost $\left(T_{2}\right)$, application of Lombricompost $\left(\mathrm{T}_{3}\right)$ and application of fresh bovinaza in liquid condition $\left(\mathrm{T}_{4}\right)$. Variables evaluated were: moisture content, bulk density, total porosity, infiltration, organic matter content, $\mathrm{pH}$ and cation exchange capacity. The results show that the fertilization with Lombricompost produce favorable effects on the physical and chemical properties evaluated, while the application of chemical fertilizers tend to produce adverse effects in these properties which can lead to a progressive deterioration of soil resource. Moreover grass Angleton, the characteristic of its root system helps improve the physical and chemical properties of soil. In most of the properties evaluated with no significant differences for a $p$ $<0.05$ in a record time of seven months, except for the infiltration and the basic cation exchange capacity.

Key words: fertilization, physical-chemical properties, organic fertilizers, Dichanthium aristatum.

\section{Introducción}

El clima de la región se clasifica como típicamente tropical con influencia de los vientos alisios que predominan durante algunos meses del año (diciembre a marzo), determinando variaciones en humedad relativa y precipitación; los vientos provenientes del noroeste se acompañan con los provenientes del norte; estas condiciones climáticas determinan tres zonas de vida de acuerdo a la clasificación de Holdridge (HOLDRIDGE, 1967): bosque seco pre-montano (ALCALDÍA DE TOLU SUCRE, 2012).

Por lo general, los suelos tropicales tienden a ser cada vez más pobres en nutrimentos, comparados con los de las zonas templadas, debido principalmente a las condiciones climáticas prevalecientes, como alta temperatura y alta humedad que aceleran los procesos de descomposición de minerales, materia orgánica y lixiviación o lavado de los nutrimentos de las plantas (CEPPI $S$ y VELASCO, 1995; LABRADOR, 2001; JARAMILLO, 2002;).

Para obtener los mejores resultados de un programa de fertilización de pastos, se debe tener en cuenta factores relacionados con el suelo, el clima y la planta, y para ello es importante la comparación entre los fertilizantes químicos y orgánicos con respecto a su efecto en las propiedades físicas y químicas de los suelos. (ICA, 1980; ICA, 1984; ICA, 1992; CEPPI S y VELASCO,. 1995; JARAMILLO, 2002). 
Con el fin de contribuir con el buen uso y manejo del recurso suelo en los sistemas de explotación ganadera de la región, que conlleve a un equilibrio entre la productividad y sostenibilidad ambiental, se desarrolló este trabajo de investigación en el período comprendido entre los meses de abril y noviembre, cuyo objetivo fue evaluar los efectos de la fertilización química y orgánica, sobre algunas propiedades físicas y químicas de un suelo con características vérticas de la Hacienda Casanare, corregimiento de Pita Abajo (Tolú).

\section{Materiales y métodos}

La presente investigación se llevó a cabo en la Hacienda Casanare, localizada en el corregimiento Pita Abajo, el cual se encuentra a $10 \mathrm{Km}$ de la cabecera del municipio de Tolú, que está ubicado al noroeste del departamento de Sucre, en las coordenadas de latitud norte $09^{\circ} 21^{\prime} 00^{\prime \prime}, 09^{\circ} 40^{\prime} 00^{\prime \prime}$ y de longitud oeste $75^{\circ} 26^{\prime}$ $00^{\prime \prime}$ y $75^{\circ} 44^{\prime} 10^{\prime \prime}$, a una altitud de 3 m.s.n.m; presenta una temperatura promedio de $28^{\circ} \mathrm{C}$, precipitación anual de $900 \mathrm{~mm}$ y $1200 \mathrm{~mm}$ (ALCALDIA DE SANTIAGO DE TOLÙ, 2012).

Los suelos del área estudiada se caracterizan por sufrir inundaciones, ya que el drenaje interno es lento a muy lento, presentándose encharcamientos de aguas lluvias, por ser suelos planos y arcillosos en su gran mayoría. El material parental está formado por aluviones, depósitos lacustres y marinos, generalmente pesados; ciertos suelos, sobre todo los cercanos al mar, presentan salinidad ligera especialmente en el subsuelo. El perfil de estos suelos es de textura arcillosa hasta $45 \mathrm{~cm}$ y siguen horizontes franco arcillosos y luego arcillo arenosos; el porcentaje de limos generalmente es alto y algunos perfiles pueden dar horizontes franco-arcillo limosos (MALAGON-CASTRO, 1983). La vegetación corresponde a la de bosque muy seco tropical, características determinadas por la influencia directa del clima.

Se realizó un reconocimiento del área de estudio para identificar el sitio de la investigación, seleccionándose un área representativa correspondiente a una (1) hectárea cultivada de Ángleton ( $D$ aristatum) en un suelo con características vérticas (MALAGON-CASTRO, 1983).

Para la evaluación de los tratamientos se utilizó un diseño completamente al azar, con cinco tratamientos y tres repeticiones, empleándose parcelas de $10 \mathrm{~m}$ de largo 
por $5 \mathrm{~m}$ de ancho, con espacios de $1.5 \mathrm{~m}$ entre las mismas las cuales correspondieron a:

Testigo $\left(T_{0}\right)$ : Sin fertilizantes

Tratamiento $1\left(T_{1}\right)$ : $\quad$ Fertilizante químico: (Urea)

Tratamiento $2\left(\mathrm{~T}_{2}\right)$ : $\quad$ Urea + Lombricompost.

Tratamiento $3\left(\mathrm{~T}_{3}\right)$ : $\quad$ Lombricompost

Tratamiento $4\left(T_{4}\right)$ : $\quad$ Bioabono (bovinaza fresca diluida en la relación 1:5 bovinaza: agua).

Inicialmente se hizo una descripción del perfil de suelo (FAO, 2006) en el lugar seleccionado y se tomaron muestras de suelo indisturbadas y disturbadas a una profundidad de 0 a $20 \mathrm{~cm}$, para sus respectivos análisis en laboratorio, de las variables humedad, densidad aparente, porosidad total, materia orgánica, $\mathrm{pH}$ y capacidad de intercambio catiónico. Además se determinó la infiltración del suelo.

De acuerdo a los resultados de caracterización físico química de las muestras iníciales de suelos, y requerimientos nutricionales del cultivo, se determinó el tipo y cantidades de fertilizantes a utilizar, haciéndose aplicaciones incorporadas en el suelo a $7 \mathrm{~cm}$ de profundidad, y distribuidas en cuatro etapas durante el transcurso de la investigación. En la primera, se aplicó a cada repetición $0,5 \mathrm{~kg}$ de Urea para $\mathrm{T}_{1}$ y $\mathrm{T}_{2} ; 130 \mathrm{~kg}$ de abono orgánico para $\mathrm{T}_{3}$, y $65 \mathrm{~kg}$ para $\mathrm{T}_{2}$, y $54 \mathrm{~L}$ de bioabono para $\mathrm{T}_{4}$; en la segunda, tercera y cuarta etapa se aplicaron en cada una, $0.25 \mathrm{~kg}$ de urea para $T_{1}$ y $T_{2}$, y $54 \mathrm{~L}$ de bioabono para $T_{4}$. Antes de iniciar las aplicaciones, en cada tratamiento se adicionó hidróxido de calcio como corrector de $\mathrm{pH}$, excepto en el tratamiento $\mathrm{T}_{0}$.

A los seis meses de la aplicación de los fertilizantes, se tomaron nuevamente muestras de suelo en cada una de los tratamientos, para su respectivo análisis, y se realizaron las pruebas de infiltración finales en cada una de éstos. Los análisis de laboratorios fueron procesados en el laboratorio de Suelos y Aguas de la Universidad de Sucre, aplicando normas técnicas colombianas de calidad (IGAC, 2006), y para las pruebas de infiltración se utilizó el método de infiltración de carga constante (IGAC, 2006).

Para comparar el efecto de los tratamientos sobre las variables físicas y químicas anotadas, se aplicó un Análisis de varianza (ANAVA), y en los casos donde se 
presentaron diferencias estadísticas significativas, se realizó la prueba de rango múltiple de Duncan, y con base en ello se hizo la interpretación de los resultados.

\section{Resultados}

Al analizar los valores medios de las variables físicas: contenido de Humedad del suelo, Densidad aparente y Porosidad total, no se presentaron diferencias estadísticas significativas a $p<0.05$, entre tratamientos para el contenido de humedad, aunque los tratamientos $T_{3}, T_{2}$ y $T_{4}$ mostraron los más altos contenidos, correspondiendo el mayor valor al Lombricompost $\left(T_{3}\right)$. En cuanto a la densidad aparente y porosidad total, no se presentaron diferencias significativas, para un $p<0.05$, entre tratamientos en ambas propiedades, aún cuando la Da. Menor se dio en el $T_{3}$. En lo referente a la Infiltración, en el tratamiento Lombricompost $\left(T_{3}\right)$ se dio la mayor infiltración, presentando al nivel de $p<0.05$, diferencia significativa con el tratamientos $\mathrm{T}_{0}$. (Fig. 1).

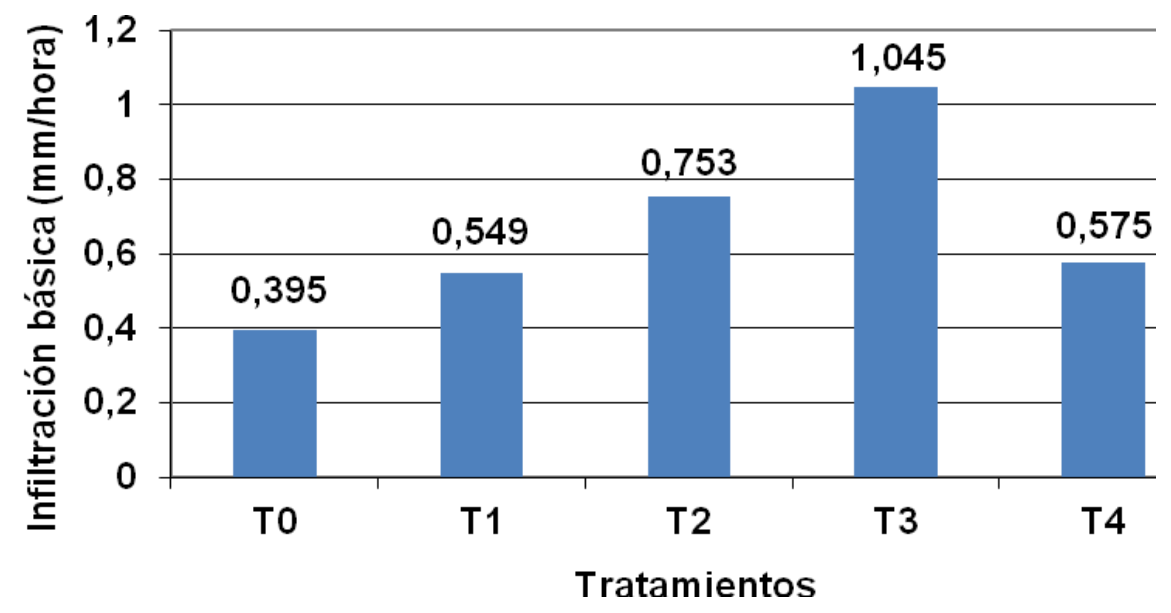

Figura 1. Efecto de diferentes tratamientos sobre la infiltración

Referente a los efectos de los tratamientos sobre las variables químicas, en la Materia Orgánica, no se presentaron diferencias significativas entre los tratamientos, sin embargo el valor más alto lo presentó el $T_{3}$ (Lombricompost), y el menor contenido se observó en el $\mathrm{T}_{4}$ (Bovinaza fresca en estado líquido). 
Respecto a la variable $\mathrm{pH}$, las mejores condiciones se dieron en los tratamientos $\mathrm{T}_{3}$ y $\mathrm{T}_{4}$ que presentaron una reacción ligeramente ácida, no presentándose diferencia estadística entre ellos, pero sí de éstos con los demás tratamientos, al nivel de $\mathrm{p}<0.05$ ( Fig. 2).

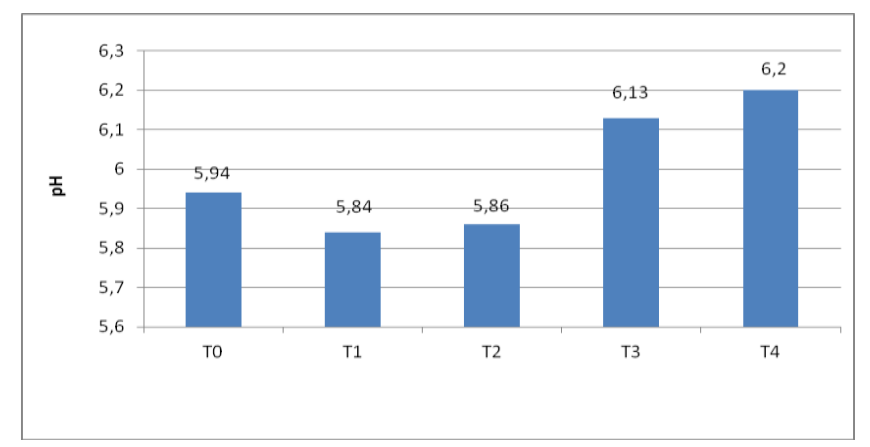

Figura 2. Efecto de diferentes tratamientos sobre el pH del suelo.

En la variable Capacidad de intercambio catiónico - CIC, para un $p<0.05$, no se presentaron diferencias significativas entre tratamientos, sin embargo el valor más alto lo presentó el tratamiento abono orgánico sólido $\left(\mathrm{T}_{3}\right)$ (Fig. 3).

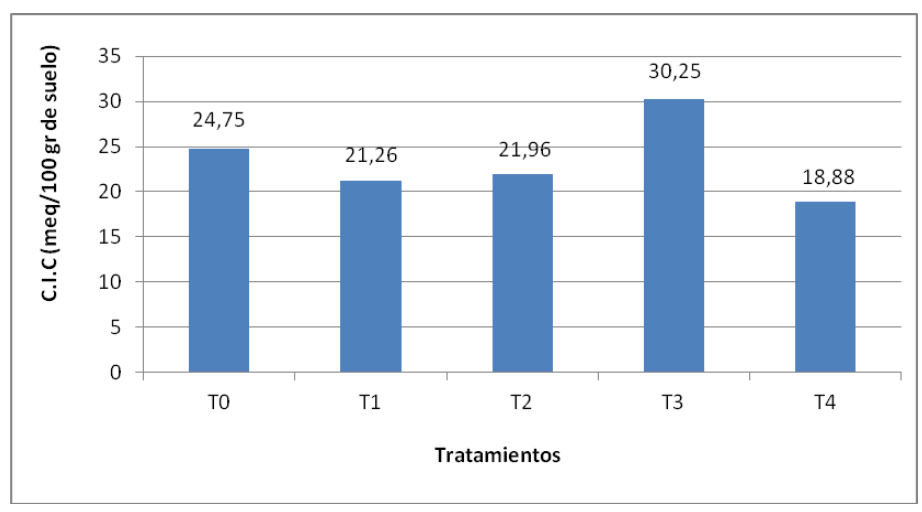

Figura 3. Efecto de diferentes tratamientos sobre la capacidad de intercambio catiónico (C.I.C.) del suelo

\section{Discusión}

Aunque en el efecto de los tratamientos sobre las variables físicas edáficas, solamente se dio diferencia estadística significativa entre el tratamiento Lombicompost $\left(T_{3}\right)$ y el testigo $\left(T_{0}\right)$ en la variable infiltración, el tratamiento $T_{3}$ 
mostró las mejores condiciones físicas edáficas, lo que está acorde con PÉREZ (2011); DURÁN y HENRÍQUEZ (2007); FÉLIX, et al. (2008).

Respecto a la variable $\mathrm{pH}$, las mejores condiciones se dieron en los tratamientos $\mathrm{T}_{3}$ y $\mathrm{T}_{4}$ que presentaron una reacción ligeramente ácida, no presentándose diferencia estadística entre ellos, pero sí de éstos con los demás tratamientos, al nivel de $p<0.05$ ( Figura 3). Al analizar la respuesta de esta variable, se puede observar que los valores de $\mathrm{pH}$ en las parcelas donde se aplicó lombricompost en su totalidad y bovinaza fresca en estado liquido se han elevado con respecto al suelo testigo. Este incremento en el pH coincide con lo reportado por OMALICO (1984) en ecosistema de pastos tropicales. TESTER (1990); LUNGU et al. (1993) y DURÁN y HENRÍQUEZ (2007) han señalado que la aplicación de estiércol y lombricompost eleva el $\mathrm{pH}$ de los suelos por los niveles de bases cambiables que estos presentan, principalmente calcio y magnesio. Igualmente coincide con los resultados reportados por OLIVIER y BORNEMISZA (1990), quienes concluyen que la aplicación de residuos orgánicos eleva el pH de los suelos. PINKULLI y ALLMARAS (1986) señalan que el aumento del pH se explica por el incremento en el contenido de carbono orgánico, que actúa como un regulador inactivando hidrógeno.

En la variable Capacidad de intercambio catiónico - CIC, el valor más alto lo presentó el tratamiento abono orgánico sólido $\left(\mathrm{T}_{3}\right)$, lo cual se debe principalmente a que en este tratamiento el contenido de materia orgánica fue el mayor y como se sabe las sustancia húmicas presentes en esta, gracias a sus numerosos radicales funcionales $\mathrm{COOH}^{-}$y $\mathrm{OH}^{-}$sobre todo, tienen una alta capacidad de cambio, con lo cual aumenta la capacidad del suelo para retener nutrientes catiónicos e intercambiarlos con la solución del mismo, además todos los compuestos orgánicos tienen superficies específicas grandes lo que conlleva al fortalecimiento del sistema de retención de nutrimentos. La CIC del suelo se ve afectada positivamente cuando se aplica un compost que esté bien maduro, como es el caso del lombricompost. Lo anterior, está directamente relacionado está directamente relacionado con el grado de humificación de la Materia Orgánica ya que el proceso de humificación produce grupos funcionales que incrementan el valor de la CIC. (ROLETTO et al., 1985; CEPPI S y VELASCO ,1995; CEGARRA et al., 1998).

En cuanto al Contenido de humedad (\%) natural del suelo en cada uno de los tratamientos, en cuanto al análisis de varianza los resultados no presentaron un 
nivel de significancia estadística para $p<0.05$, entre los tratamientos, pero a pesar de esto se puede apreciar que la aplicación de abono orgánico sólido aumentó los valores de humedad natural en el suelo, lo que coincide con lo señalado por CEPPI S y VELASCO (1995); BLANCO (2002).

Para el caso de la Infiltración básica ( $\mathrm{mm} / \mathrm{hora}$ ), los valores de infiltración básica obtenidos en campo, fueron mayor en $T_{3}$ y menor en $T_{0}$. El análisis de la varianza de los valores de la infiltración básica en los tratamientos arrojó un nivel de significancía para un $p<0.05$, presentándose además una diferencia entre los tratamientos (Prueba de Duncan); siendo probable que el valor más alto presentado en $\left(T_{3}\right)$ se deba a la aplicación de abono orgánico sólido que se hizo en este tratamiento, ya que según estudios realizados mejora la tasa de infiltración del agua en muchos suelos, especialmente los de textura fina, las razones de este efecto son muchas e incluye el aumento de la agregación de las partículas del suelo, que aumenta el tamaño promedio de los poros de la rizosfera, además la estabilidad de los agregados superficiales aumenta en suelos que reciben abonos orgánicos y por ende mejora la permeabilidad y la infiltración del agua en el suelo (CEPPI y VELASCO , 1995). Por otra parte la incorporación de abono orgánico en el suelo favorece la presencia de macroorganismos como la lombriz de tierra la cual según el SOIL QUALITY INSTITUTE (SQI, 1999), y CEPPI S B, VELASCO M. 1995, la adsorción de ácidos húmicos sobre partículas de suelo mejora la calidad del suelo debido a que afectan positivamente la agregación y la porosidad.

En el caso de $T_{2}$ (fertilización química mas abono orgánico sólido) en el cual se aplicó en mayor proporción el abono orgánico con relación al fertilizante químico aplicado, se refleja de igual manera la incidencia de la materia orgánica en la infiltración del suelo. Los efectos de la aplicación de fertilizante químico $\left(T_{1}\right)$ en el suelo se ven reflejados en una disminución de la infiltración, debido a que estos por lo general producen una disminución de $\mathrm{pH}$ en el suelo desfavoreciendo el trabajo de las bacterias, las cuales en condiciones ácidas no descomponen a buen ritmo los residuos orgánicos provocando una disminución en los contenidos de materia orgánica en el suelo, lo que repercute de forma negativa en algunas propiedades físicas del mismo, tales como la infiltración (CEGARRA et al., 1998; SOIL QUALITY INSTITUTE- SQI, 1999; LABRADOR, 2001; BLANCO, 2002).

Para el caso de la materia orgánica (\%) en promedio para cada uno de los tratamientos y sus repeticiones, el mayor incremento se presentó en el tratamiento $T_{3}$; debido al alto contenido de materia orgánica presente en el 
Lombricompost (11.12\%), según LABRADOR, M (2001) y por otra parte el estiércol fresco se caracteriza por tener un alto contenido de microorganismos y una alta relación C/N, BLANCO (2002); DURÁN y HENRÍQUEZ, 2007.

\section{Conclusiones}

El tratamiento Lombricompost $\left(T_{3}\right)$, con una dosis de 2.6 t/ha, presentó a los 6 meses de aplicación del abono las mejores condiciones físicas y químicas edáficas: mostró el más alto contenido de humedad; se dio en él la mayor infiltración; presentó el valor más alto de materia orgánica (MO); se dio una buena condición del pH para la explotación de cultivos; se dio la mayor capacidad de intercambio catiónico CIC; y una de la más baja Densidad aparente, después del $\mathrm{T}_{4}$.

En el $T_{0}$ se dio el menor contenido de humedad (\%), y la menor infiltración; en $T_{1}$ se encontró la mayor acidez (menor $\mathrm{pH}$ ), una baja infiltración y una baja CIC, después del $\mathrm{T}_{4}$; en el $\mathrm{T}_{2}$, se encontró un buen \% de humedad, y mayor CIC que los $T_{4}$ y $T_{1}$. El tratamiento $T_{4}$ (bovinaza líquida) mostró el menor contenido de materia orgánica y la más baja capacidad de intercambio catiónico $\mathrm{CIC}$, y la más baja Densidad aparente; pero el más alto $\mathrm{pH}$, favorable para los cultivos en ese agroecosistema.

\section{Referencias}

ALCALDIA MUNICIPAL DE SANTIAGO TOLU. 2012 - 2015. Plan de Desarrollo Municipal "la oportunidad para todos". Tolú- Sucre.

BECERRA, B.J.; AVENDAÑO, M.J. 1992. Efecto de la severidad de defoliación sobre la producción de forraje y los carbohidratos de reserva en especies tropicales. REVISTA Téc Pecu Méx. 30(2):125-132

BLANCO S. ICA. Acondicionadores y mejoradores de suelo. [Serial online]. 2002. [Citado 19 de Nov. 2002]; 1 (1) [28 pantallas]. Disponible en: URL: http://www.agronet.gov.co/www/docs si2/2006718153746 Acondicionadores\%20y \%20mejoradores\%20de\%20suelo.pdf

CEGARRA, M.I.; RAGONA, D.; GARCI-ESPINA, R.; GONZALEZ, P.D.; LO FORTE, G.L.; SATO, A.M., 1998. . Factores que influyen en la extracción y fraccionamiento de la materia orgánica del suelo. Revista Anales de Edafología y Agrobiología 33:575-590. 
CEPPI S, B.; VELASCO, M. 1995. Adsorción de ácidos húmicos sobre partículas de suelo: Influencia del desarrollo de cargas superficiales y de cationes. Revista Bol.Soc. Chile. Quim. 40:197-205

DURÁN, L.; HENRÍQUEZ, C. 2007. Caracterización química, física y microbiológica de vermicompostes. Revista Agronomía Costarricense 31(1):41-51.

FAO. 2006. La Nueva Guía para la Descripción del Perfil de suelo de la FAO. Roma.

FÉLIX, J.; SAÑUDO, R.; ROJO, G.; MARTíNEZ, R.; OLADE, V. 2008. Importancia de los abonos orgánicos. Revista Ra Ximhay 4:57-67.

HOLDRIDGE, L.R. 1967. Determination of World Plant Formations from Simple Climatic Data. Science 105 (2727):367-368.

INSTITUTO COLOMBIANO AGROPECUARIO ICA. 1992. Los suelos y su fertilidad. Bogotá, Colombia.

INSTITUTO COLOMBIANO AGROPECUARIO ICA. 1984. Fertilización de cultivos en clima cálido. Edición No. 1. Bogotá, Colombia.

INSTITUTO COLOMBIANO AGROPECUARIO ICA.1980. Suelos y fertilización de cultivos. Compendio No 38. Bogotá, Colombia.

INSTITUTO GEOGRÁFICO AGUSTíN CODAZZI. 2006. Métodos Analíticos del laboratorio de suelos. 6 edición. Bogotá, Colombia.

INSTITUTO GEOGRÁFICO AGUSTíN CODAZZI. 1998. Estudio General de Suelos y Zonificación de Tierras: Departamento de Sucre. Santa fe de Bogotá:

JARAMILLO, J.; DANIEL, F. 2002. Introducción a la Ciencia del Suelo. Universidad. Nacional de Colombia. Facultad de Ciencias. Medellín.

LABRADOR-MORENO, J. 2001. La Materia orgánica en los ecosistemas. Ediciones Mundi Prensa. Madrid, España.

LUNGU, O.I.; TEMBA, B.; LUNGU, C. 1993 Efects of lime and farmyard manure on soil acidity and maize growth on acid Alfisol from Zambia. Revista Trop. Agric. 4:309-314.

MALAGON C,D. 1983. Estudio general de suelos de la región noroccidental del departamento de Sucre. Instituto Geográfico Agustín Codazzi. Bogotá, Colombia. 
MALAGON C,D.; MONTENEGRO G,H. 1990. Propiedades físicas de los suelos. Instituto Geográfico Agustín Codazzi IGAC. Bogotá, Colombia.

OLIVIER R. C. y BORNEMISZA. E.1.990. Efecto de residuos orgánicos y abonamiento mineral sobre las propiedades químicas de un Typic Humitropept en Turrialba Costa Rica. Revista Agronomía costarricense. 14: 237-240.

OMALIKO, C.P. 1984. Dung decomposition and its effects on the soil component of a tropical grassland ecosystem. Revista Trop. Ecol. 25:214-220.

ORGANIZACIÓN DE LAS NACIONES UNIDAS PARA LA AGRICULTURA Y LA ALIMENTACIÓN “FAO”. 2009. Guía Para La Descripción De Suelo. Roma.

PLAN DE DESARROLLO MUNICIPAL 2012 - 2015. Región Golfo de Morrosquillo y municipio de Tolú. p $58-89$.

PÉREZ CARDOZO R. 2011. Efecto de tres abonos orgánicos en el agroecosistema de Bothrichloa pertusa, (L) A. Camus en fincas ganaderas de Sampués, Sucre-Colombia. [Tesis de Maestría].Sincelejo, Colombia: Sistema Universitario Estatal del Caribe (Sue-Caribe), Universidad de Sucre, Facultad de Ciencias Agropecuarias.

PIKULL, J.L. Y R.R. ALLMARAS. 1986. Physical and chemical properties of a haploxeroll after fifty years of residue management. Revista Soil Sci. Soc. Am. J. 52:214-219.

ROLETTO, E., R. BARBERIS, M. CONSIGLIO, and R. JODICE. 1985. Che33mical parameters maturity. Revista BioCycle, 26: 46-47.

SQI-SOIL QUALITY INSTITUTE. 1999. Indicators for Soil Quality Evaluation. USDA Natural Resources Conservation Service. Prepared by the National Soil Survey Center in cooperation with The Soil Quality Institute, NRCS, USDA, and the National Soil Tilth Laboratory, Agricultural Research Service. USA.

TESTER, C.F.1990. Organic amendments effects on physical and chemical properties of a sandy Soil. Revista Soil Sci. Soc. Am. J. 54: 827-831. 